\title{
Feelings of Social Isolation in Canada's Pandemic Times: Exploring Its Correlates Using Multiple Correspondence Analysis ${ }^{1}$
}

Fernando Mata

School of Sociology and Anthropology

University of Ottawa

fmata@uottawa.ca

July 6, 2021

\begin{abstract}
Identifying the linkages between the feelings of social isolation and its socio-demographic, residential, and psychosocial correlates is of major importance to the healthcare system today. Using data from the Canadian Survey Perspective Survey Series 6 (CPSS6) conducted by Statistics Canada, the purpose of this study is to explore these linkages during the second year of the pandemic in Canada. Five levels of social isolation were examined in conjunction with 17 other socio-demographic and psychosocial characteristics of 3,941 respondents to the survey. The study suggests that about three out of four Canadians (75\%) have experienced some feeling of social isolation at some point during the pandemic. The most severe form comprised $11 \%$ of the total population. In the context of a generalized cross-tabular data analysis, Multiple Correspondence Analysis (MCA) detected two major dimensions underlying the data: mental health distress and time duration. These dimensions represented approximately $61 \%$ of the inertia or unexplained variation in the data. An examination of the combinations of variable categories revealed that the highest perceived isolation levels were found among young individuals, females, individuals who were single, those who received medical help during the pandemic, and those living in low-rise apartments in urban areas. The lowest levels of perceived social isolation were found among older individuals, rural residents, those who were married and/or reported excellent mental health. Not all individuals who experienced higher levels of mental distress, however, felt social isolation. The findings of the study could be useful when designing public health campaigns aimed at reducing social isolation by providing helpful alternatives to affected populations.
\end{abstract}

Keywords: Feelings of Social Isolation, Canada, Pandemic, Multiple Correspondence Analysis

\subsection{Introduction}

In the social psychology literature, feelings of social isolation are conceptualized in many ways which vary according to the particular theoretical frameworks used by researchers. There is an agreement, however, that it refers to an evaluation of the quality of interactions that an individual has with the rest of society. Socially isolated individuals report having poor or limited contact with others and/or assess that this contact is either inadequate or insufficient (Findlay, 2003). In many cases, like in the case of epidemics, this absence of or inadequate contact is perceived as having adverse personal consequences (e.g., contracting viruses and getting sick). The concept of social isolation can also be distinguished from that of loneliness which often appears to be associated with a voluntary decision to live apart from others (Van Baarsten, et al., 2001). Factors associated with feelings of social isolation include a sense of personal "loss", poor physical and mental health

\footnotetext{
${ }^{1}$ Paper to be presented at the CSA Meetings, University of Victoria, B.C, June 4-8, 2022. The author would like to thank CERC at Ryerson University, the Ontario Data Documentation, Extraction Service and Infrastructure (ODESI-Scholars Portal Statistics) as well as Statistics Canada for making available the data and valuable support and guidance.
} 
as well as other contextual elements such as geographic location, available communication vehicles and/or means of transportation. Evidence from various studies also suggests that adults who feel socially isolated experience higher levels of anxiety and stress as well as lower levels of optimism, happiness, and life satisfaction (see, for instance, Cacciopo and Patrick, 2008).

Before the start of the COVID-19 pandemic, studies have also shown that some population segments such as older adults, people with disabilities, the homeless, those living alone, and/or who had pre-existing health conditions were among the most likely to report feelings of being socially isolated. In their efforts to contain the virus, the COVID-19 lockdowns have led to home curfews, restrictions on the assembly of groups, cancellation of planned social and public events, closure of mass transit systems, and other travel restrictions (Hwang et. al, 2020). By limiting human interactions to only household "bubbles"2, pandemic lockdowns have worsened the mental health quality of affected populations. In Canada, for instance, there is mounting evidence that various segments of the Canadian population are experiencing deterioration in terms of their mental health (Jenkins. et. al, 2021). Those who experienced health, social, and/or structural vulnerabilities due to pre-existing mental health conditions, disability, income, ethnicity, sexuality, and/or gender are said to be among those more likely to report mental health deterioration and difficulties coping with the pandemic.

Using Statistics Canada's Canadian Perspective Survey Series (CPSS) data collected in early 2021, this study aims at increasing our knowledge on the subject by focusing its attention on the feelings of social isolation experienced during the pandemic period in Canada. Two major research goals are pursued here: 1) to empirically explore the association between social isolation and its socio-demographic, residential, and psychosocial correlates to obtain an overall picture of this phenomenon in Canada, and 2) to examine the nature of the major dimensions underlying the survey data. The reader should note that the study is exploratory and is aimed at detecting data patterns rather than testing predictive models with the data at hand.

\subsection{Data Source, Methodological Approach and Measures}

The data for this analysis is drawn from the Canadian Perspectives Survey Series 6 survey (CPSS6) entitled "Substance Use and Stigma During the Pandemic". This online survey collected information about the use of various prescription and non-prescription substances. The probability panel for the CPSS6 survey was created by randomly selecting a subset of the larger Labour Force Survey (LFS). The survey was conducted by Statistics Canada between January 25-31, 2021, and collected information on approximately 4,941 Canadians aged 15 years old and over who were residents of the 10 Canadian provinces.

The methodological approach to analyze the data is based on an exploratory socio-ecological perspective of health outcomes (Grzywacz and Fuqua, 2000). According to this perspective, different dimensions of wellbeing are linked to diverse conditions present in the socio-physical environment. The feelings of social isolation are seen in the wider context of other individual psychosocial, residential and demographic factors which affect its development and present configuration.

The central variable of interest, the feelings of being socially isolated during the pandemic, was measured by five levels which were reported answering the following question: In general, how often have you felt the following since the start of the COVID-19 pandemic? with categories: $1=$ Never, 2=Hardly Ever, $3=$ Some of the time, $4=$ Often, $5=$ Always. This variable is treated as a nominal variable and is not aggregated into binary categories to capture finer levels of detail ${ }^{3}$.

\footnotetext{
${ }^{2} \mathrm{~A}$ "bubble" is an unofficial term used in Canada to describe people with whom the individual feels comfortable spending time during the pandemic (e.g., the household).

${ }^{3}$ Other analytical possibilities included treating social isolation (5 levels) as an ordinal and even a continuous variable. These assumptions would have led to the development of more traditional ordinal probit, logistic, and/or multinomial models. However, within a more exploratory context, the choice of the
} 
Demographic correlates of social isolation ( $\mathrm{n}=8$ variables) included: age, gender, marital status, immigrant status, a child under 18 living with the respondent (yes, no), the highest level of education completed, employment status, and the number of household members where the respondent lived. Residential correlates ( $\mathrm{n}=2$ variables) included the area of residence (urban, rural) and type of dwelling where the respondent lived.

Psychosocial correlates ( $\mathrm{n}=7$ variables), included the following:

(1) People to talk to, with the question phrasing: Approximately how many relatives and friends do you have who you feel close to, that is, who you feel at ease with and can talk to about what is on your mind? Categories: $1=$ None $2=1$ or more

(2) Lack of companionship with question phrasing: In general, how often have you felt a lack of companionship since the start of the COVID-19 pandemic? Categories: 1=Never, 2=Hardly Ever, $3=$ Some of the time, $4=$ Often, $5=$ Always

(3) Feeling left out with question phrasing: In general, how often have you felt feeling left out since the start of the COVID-19 pandemic? Categories: 1=Never, 2=Hardly Ever, 3=Some of the time, $4=$ Often, 5=Always;

(4) Reported pandemic stress with question phrasing: In general, how would you describe the amount of stress in your life, since the start of the COVID-19 pandemic? Categories 1=Low Stress, 2=High Stress

(5) Life Satisfaction level with question phrasing: Using a scale of 0 to 10, where 0 means "very dissatisfied" and 10 means "very satisfied", how do you feel about your life as a whole right now? Categories: Low (Under 5 points), Moderate (5-7 points), High (8 points or higher)

(6) Perceived mental health with question phrasing: In general, how would you describe your mental health? Categories: 1=Excellent, 2=Very Good,3= Good, 4=Fair, 5=Poor

(7) Support received during the pandemic with question phrasing; Throughout the COVID-19 pandemic, have you ever seen, or talked on the telephone to, a medical doctor, family \&friends or other individuals (yes, no)? Categories: $1=$ Yes Medical, $2=Y e s$, Family\& Friends $3=Y e s$, Others, $4=$ No support received.

Given that all variables collected by the CPSS6 are treated as categorical, they can be jointly examined in the context of a generalized cross-tabular analysis. Multiple Correspondence Analysis (MCA) is an ideal multivariate statistical tool suited for this purpose as it effectively visualizes relationships where the positions of variable categories are mapped into a simpler dimensional plot called bi-plot (Greenacre, 2010). MCAs extract data dimensions that account for different portions of data variability called "inertia" (Everitt and Dunn, 2001). The contributions of particular variable categories to "inertia" are assessed by the square cosines of angles $(\theta$ 's) formed by the variable vectors and those representing the major dimensions axes. In the context of MCA analysis, particular combinations of variable categories reflect the positions of various population segments within the major data dimensions.

\subsection{Findings}

\subsection{General Picture}

According to the CPSS6 (see Chart 1), about two-thirds of the Canadian population (64\%) reported that they experienced social isolation either sometimes or often during the pandemic period in Canada. This population represents about 19.8 million individuals. Those who always felt isolated during the pandemic represented $11 \%$ (3.4 Million) while those hardly ever or who did not experience isolation represented $25 \%$ of the total population (7.6 Million). Chart 2 displays a more detailed breakdown of these isolation levels by age-gender groups. Social isolation was experienced more intensely by younger individuals (under 35 years old). One in five females $(20 \%)$ of this population felt very isolated while $16 \%$ of males did as well. More than one-third of these individuals also reported that they often felt socially isolated $(34 \%$ and

statistical tool was guided by the criteria of the visualization of results in a geometrical plane where personal attributes that are situated nearby reveal the strength of associations between variables. 
$35 \%$ respectively).

Chart 1: Feelings of Social Isolation* experienced during the COVID-19 pandemic, Canada 2021

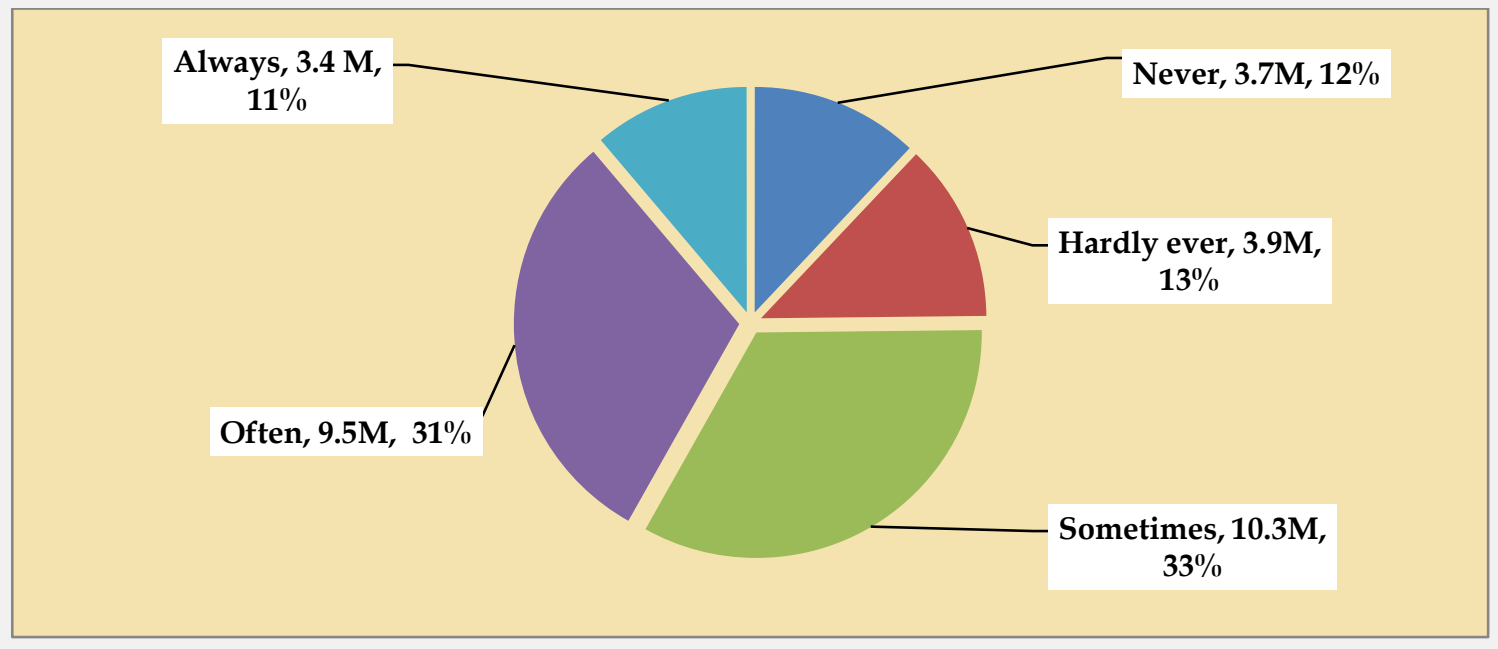

* Weighted population counts (in millions). Source: CPSS6 Survey, Statistics Canada, 2021

Chart 2: Feelings of Social Isolation* experienced during the COVID-19 pandemic, Age-Gender groups, Canada 2021

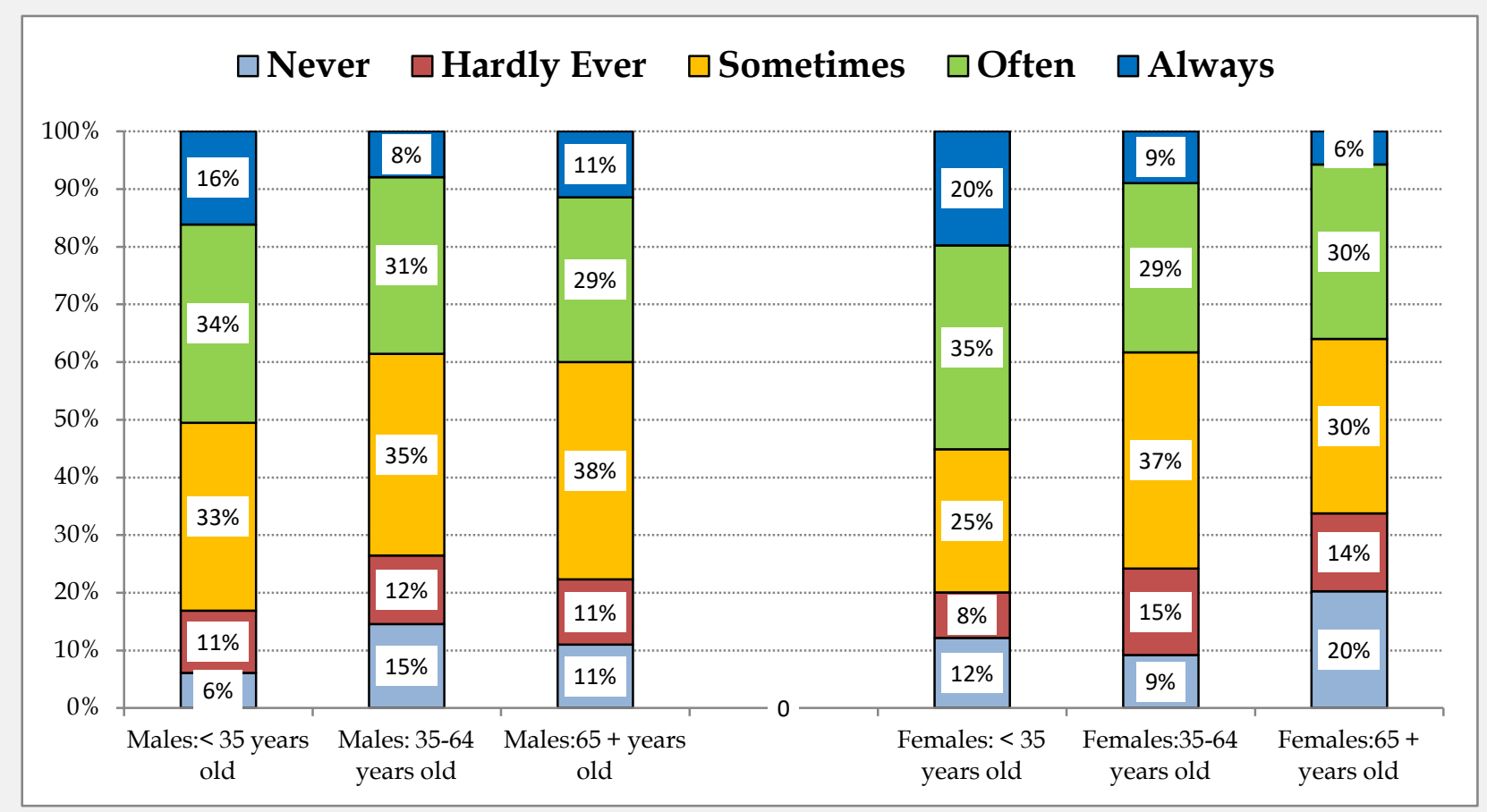

Source: CPSS6 Survey, Statistics Canada, 2021 
To expand the range of descriptive explorations of the data, binary correlations ${ }^{4}$ were calculated between the five levels of isolation and variable categories of its 17 socio-demographic, residential, and psychosocial correlates. The correlations for three levels of isolation are presented in Chart 3 . The highest observed for the highest level of social isolation (category: always) were the feelings of being left out (category: always), lack of company (category: always), poor mental health, and lower life satisfaction scores ( $\mathrm{r}=+.30$ or higher). Those corresponding to the lowest level of social isolation (category: never) were the lack of company (category: never) and very good mental health ( $\mathrm{r}=+.45$ or higher).

Chart 3: Binary Correlations*: Feelings of Social Isolation and variable categories of socio-demographic, residential, and psychosocial correlates

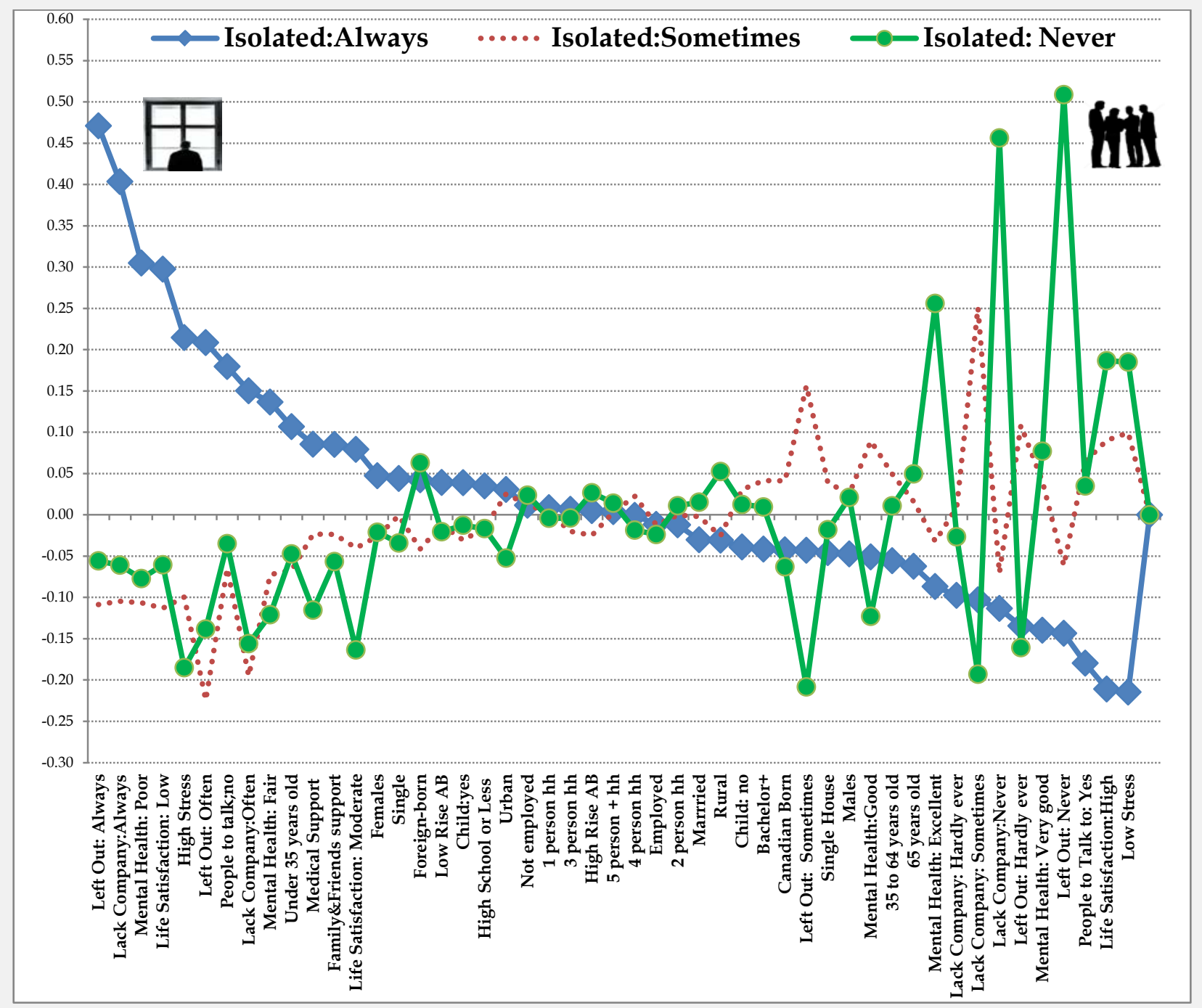

* Not all variable categories are shown. The correlation coefficient necessary to attain significance at the .05 with a sample of 3,900 is approximately r=(abs).041. Source: CPSS6 Survey, Statistics Canada 2021

\footnotetext{
4 Tetrachoric binary correlations are used here. These provide estimates if ratings were made on a continuous scale of the dependent variable which is assumed to be invariant of rated categories (Uebersax, 2015).
} 


\subsection{MCA Findings}

The first step of the explorations using MCA consisted of an overall assessment of the strength of the relationships between social isolation and its correlates. This was accomplished by undertaking a series of Chi-square tests (see table 2). All 17 correlates of social isolation were found to be statistically significant at the $\mathrm{p}<.05$ level. Psychosocial correlates such as the feelings of being "left out", lack of company, perceived mental health, life satisfaction, and reported stress levels displayed greater discriminatory power compared to socio-demographic and residential correlates.

Table 2. Chi-Square Test Results*: Socio-demographic, Residential and Psychosocial Correlates of Feelings of Social Isolation

\begin{tabular}{|c|c|c|c|c|}
\hline & Variables & TestValue & d.f & sig. \\
\hline 1 & Feeling Left Out & 2867.7 & 16 & 0.00 \\
\hline 2 & Lack of Company & 2402.5 & 16 & 0.00 \\
\hline 3 & Perceived Mental Health & 1084.2 & 16 & 0.00 \\
\hline 4 & Life Satisfaction & 752.6 & 8 & 0.00 \\
\hline 5 & Reported Stress Level & 532.3 & 4 & 0.00 \\
\hline 6 & Support Received & 288.2 & 12 & 0.00 \\
\hline 7 & People to Talk to & 137.4 & 4 & 0.00 \\
\hline 8 & Age Groups & 86.0 & 8 & 0.00 \\
\hline 9 & Immigrant Status & 30.9 & 4 & 0.00 \\
\hline 10 & Dwelling type & 29.1 & 12 & 0.00 \\
\hline 11 & Marital Status & 22.3 & 12 & 0.03 \\
\hline 12 & Urban Residence & 19.0 & 4 & 0.00 \\
\hline 13 & Educational Level & 17.4 & 8 & 0.03 \\
\hline 14 & Gender & 14.4 & 4 & 0.01 \\
\hline 15 & Living with Child Under 18 & 13.0 & 4 & 0.01 \\
\hline 16 & Employment Status & 8.3 & 4 & 0.08 \\
\hline 17 & Household Size & 7.7 & 16 & 0.04 \\
\hline
\end{tabular}

*- Chi Square Test Results Ranked by test value, Source: CPSS6 Survey, Statistics Canada, 2021

Table 3: Square Cosines* of variable categories concerning mental health distress (D1) and time duration (D2) dimensions (ranked)

\begin{tabular}{|c|c|c|c|}
\hline $\begin{array}{l}\text { Variables } \\
\text { Categories D1 } \\
\text { (Mental Health Distress) }\end{array}$ & $\operatorname{Cos}^{2} \mathrm{D} 1$ & $\begin{array}{l}\text { Variables } \\
\text { Categories D2 } \\
\text { (Time Duration) }\end{array}$ & $\operatorname{Cos}^{2} \mathrm{D} 2$ \\
\hline 1.Life Satisfaction: High & 0.383 & 1.Left Out: Never & 0.277 \\
\hline 2. High pandemic Stress & 0.351 & 2. Lack Company: Always & 0.261 \\
\hline 3.Low Pandemic Stress & 0.351 & 3.Socially Isolated: Never & 0.176 \\
\hline 4.Socially Isolated: Always & 0.251 & 4.Life Satisfaction: Low & 0.172 \\
\hline 5.Left Out: Never & 0.242 & 5.Left Out: Never & 0.168 \\
\hline 6. No support received & 0.228 & 6.Isolated: Never & 0.165 \\
\hline 7.Mental Health: Poor & 0.221 & 7.Lack Company: Never & 0.138 \\
\hline 8.Lack Company: Often & 0.213 & 8. Socially Isolated: Often & 0.128 \\
\hline 9.Left Out: Often & 0.210 & 9.Left Out: Sometimes & 0.126 \\
\hline 10.Lack Company: Never & 0.203 & 10.Mental Health: Poor & 0.124 \\
\hline 11.Life Satisfaction: Moderate & 0.185 & 11.Mental Health: Good & 0.106 \\
\hline 12.Mental Health: Fair & 0.184 & 12.Lack Company: Sometimes & 0.104 \\
\hline 13.Life Satisfaction: Low & 0.182 & 13.Mental Health: Excellent & 0.098 \\
\hline 14.Lack Company: Always & 0.170 & 14.Life Satisfaction: Moderate & 0.090 \\
\hline 15.Mental Health: Very Good & 0.169 & 15. No people to talk to & 0.069 \\
\hline 16.Left Out: Always & 0.160 & 16.People to talk to & 0.069 \\
\hline 17.Socially Isolated: Never & 0.143 & 17.Employed & 0.063 \\
\hline 18.Socially Isolated: Often & 0.119 & 18. Not Employed & 0.063 \\
\hline 19.Mental Health: Excellent & 0.115 & 19.Family\&Friends Support & 0.053 \\
\hline 20. Single marital status & 0.096 & 20. Socially Isolated: Sometimes & 0.047 \\
\hline
\end{tabular}

* Squared cosine values may be interpreted as the shared variance between the variable and the respective dimension.

Source: CPSS6 Survey, Statistics Canada, 2021 
MCA extracted two major dimensions from the CPSS6 survey data. These two dimensions were labeled Mental Health Distress (D1) and Time Duration (D2) respectively. They accounted for $61.5 \%$ of the unexplained variation in the data $(49.9 \%$ and $11.6 \%$ respectively). Table 3 shows the variable contributions to these extracted dimensions. The dimension of mental health distress has its highest contributions from the following variable categories: life satisfaction (higher scores), high and low reported pandemic stress, feeling socially isolated (category: always), and feelings of being "left out" (category: never). The dimension of time duration has its highest contributions from the following variable categories: feelings of "left out" (category: always), lacking company (category: always), feelings of being socially isolated (category: always), and lower life satisfaction scores.

The asymmetric MCA bi-plot ${ }^{5}$ displaying the positions of all variable categories in the two major dimensions axes is presented in Chart 2. These positions are presented in four quadrants (I to IV) representing combinations of low-high mental distress and short-long episode event duration respectively. About $31 \%$ of all cases were found in quadrant I (high distress, short duration), $13 \%$ in quadrant II (high distress, long duration), 20\% in quadrant III (low distress, long duration), and 36\% in quadrant IV (low distress, short duration). The plot of cases in the four quadrants reveal a "U" pattern characteristic of MCA type of plots.

The highest level of social isolation (category: always) was found in quadrant II where indicators of mental health distress and time duration are of highest magnitudes. The feeling of being "left out" is the major psychosocial driver of membership of variable categories within quadrant II. The combinations of variable categories found in the bi-plot are proxies for identifying at-risk populations. Individual characteristics such as poor mental health, low life satisfaction, lacking the company of others, having received medical help during the pandemic, and no people to talk to are typical in the quadrant. Being single, female, being part of a 1-person household, and/or living in low-rise apartments building are demographic indicators of the risk of social isolation. The MCA bi-plot also reveals that a higher level of mental distress does not necessarily correspond to a higher level of feeling social isolation. Some demographic segments appear more resilient than others. This is the case of populations corresponding to the characteristics of variable category combinations found in quadrant I. Despite experiencing high levels of distress, individuals experience social isolation in a more episodic fashion rather than constantly (category: often). Individuals corresponding to these experiences of isolation usually report moderate levels of life satisfaction, good mental health, and having received emotional support from family and friends during the pandemic.

Quadrant III presents combinations of variable categories describing favourable mental health outcomes. Populations conforming to these variable category combinations report lower levels of social isolation (category: never). Individuals describe themselves in excellent mental health, never feeling "left out" and/or lacking the company of others. Higher scores of life satisfaction are also observable as typical psychosocial traits of this demographic segment. Being older (65 years old and over), married, and/or a resident of a rural area appears to be "protective" demographic factors concerning social isolation and is associated with the most favorable health outcomes. Finally, similar to population segments represented in quadrant I, the combination of variable categories located in quadrant IV reflect the more fleeting experiences of social isolation (category: sometimes) also within the context of a lower level of mental health distress as populations of quadrant III. In terms of socio-demographic characteristics, those individuals of higher levels of education and/or who live in single-detached single houses are noteworthy to mention.

\footnotetext{
5 In bi-plots, vectors representing variable categories are located in four quadrants representing higher or lower than average values concerning the mental health distress and time duration dimensions respectively (i.e., standard deviation units). The proximity of vectors to group positions in space suggests an over-representation of these traits concerning the composition of groups while greater distances suggest its converse. Correlations between two vectors in component space are equal to the cosines of the angles between the indicator vectors $(\theta)$, or $r=\cos (\theta)$. Highly $\operatorname{correlated}$ vectors are located at sharp angles from each other $(\theta=90$ degrees or less) while those zero correlated are "orthogonal" to each other $(\theta=90$ degrees). If variables are perfectly negatively correlated, then $\theta=360$ degrees (vector in opposite direction)
} 
Chart 2: MCA's Asymmetric Bi-plot of Variable Categories and Observations: Social Isolation and Its Socio-demographic, Residential and Psychosocial Correlates*

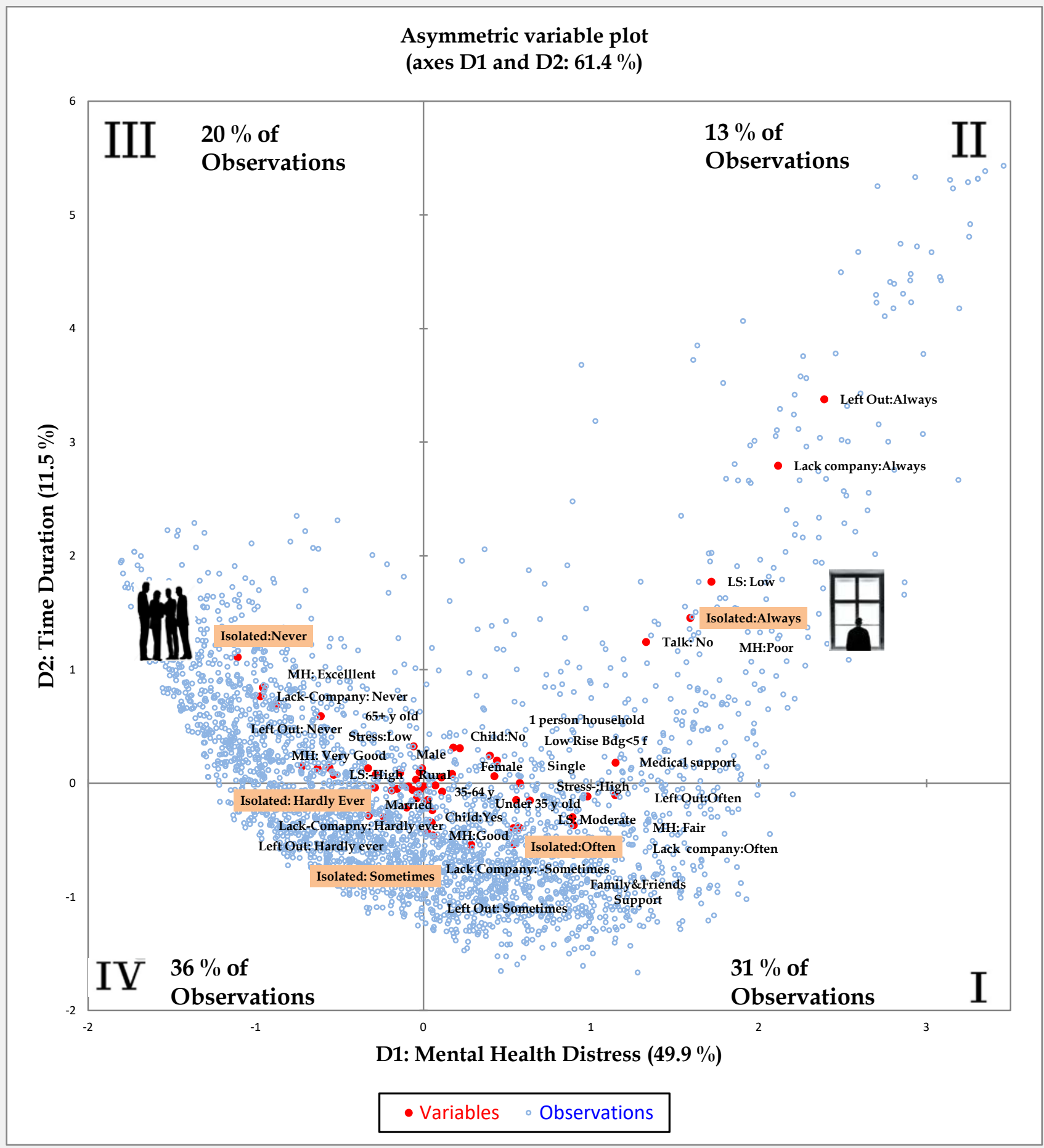

* Not all variable categories are shown. Symbols: MH=Mental Health, LS=Life Satisfaction, Talk: People to talk to. Percentages of explained inertia shown in parentheses. Source: CPSS6 Survey, Statistics Canada 2021

Overall, MCA explorations present us with a national picture where the feelings of social isolation are part of a constellation of health outcomes characteristic of a population struggling to cope with COVID-19 
lockdowns. At the time of the survey, some segments of the Canadian population appeared more vulnerable to social isolation than others. Younger individuals, females, those who have received medical help during the pandemic and/or reside in low-rise apartment buildings appear at higher risk for social isolation. This is because their social networks have been seriously disrupted and there is a lack of emotional support drawn from them. Regardless of the comfort that communication technologies may offer them in terms of "virtual" contact, they feel "left out" and starved for the company of others. Another interesting finding is that more sporadic feelings of isolation are found among individuals of both higher and lower mental distress levels (e.g., associations found in quadrants I and IV). This finding suggests that otherwise mentally healthy individuals were not immune to episodes of feeling socially isolated at some points during the pandemic.

\subsection{Post- Explorations Reflections}

Using multiple correspondence analysis as an analytical tool, this study focused on the perceptions of social isolation and its socio-demographic, residential, and psychosocial correlates. The available public version of the CPSS6 survey, regrettably, only contained a limited number of relevant variables and did provided provincial or territorial breakdowns. The overall picture obtained during the second wave of the pandemic is likely to change in the subsequent phases of the pandemic due to vaccination progress and potential virus mutations so it is desirable to produce a larger longitudinal picture of how social isolation is experienced by the various segments of the Canadian population.

The study suggests that three out of four Canadians (75\%) have experienced some form of social isolation at some point during the pandemic. The most severe form comprised about $11 \%$ of the total population. Populations at-risk seemed to comprise young individuals, females, those who were single, those who received some form of medical help, and those living in low-rise apartments in urban areas. Protective factors identified include good mental health, being married, and rural residence. This study also shows that individuals whose overall mental health was good felt socially isolated at some point during the pandemic. Given the nature of the cross-sectional data, it is not possible to elucidate the direction of causality between social isolation and other mental health outcomes, but these connections are suspected to be strong. Another point of reflection refers to the fact that neither employment status, educational level, nor immigrant status had any visible impacts on feelings of social isolation, as one may expect. Social isolation episodes seem to cut across socio-economic and employment borders and are being felt in all corners of society in the pandemic environment of today.

Given the study results, it is important to briefly reflect on the type of health interventions that can be designed to reduce social isolation in Canada. These strategies may include communication via the most common social media vehicles utilized by various age cohorts of Canadians. Support groups offer another alternative, particularly those that contain educational or cultural elements (such as learning a hobby) and those that expand circles of friends. Community support services are also beneficial to health and well-being. The pandemic period raises also questions surrounding the role that communication technologies have in alleviating feelings of social isolation. These tools have become critical during crises situations such as the current COVID-19 emergency occurring in Canada. Through them, individuals gather necessary information, follow developments and sanitary guidelines, seek social support, and/or express anxieties about traumatic events.

\subsection{References}

Brooks, S. K., Webster, R. K., Smith, L. E., Woodland, L., Wessely, S., Greenberg, N., and Rubin, G. J. (2020). The psychological impact of quarantine and how to reduce it: Rapid review of the evidence. Lancet, 
$395,912-920$.

Cacioppo, J.T., and Patrick, W. Loneliness: Human Nature and the Need for Social Connection. (2008). New York: W.W. Norton \& Company.

Everitt, B.S., and Dunn, G. (2001). Applied Multivariate Analysis. London: Hodder Arnold.

Findlay, R. (2003). Interventions to reduce social isolation amongst older people: where is the evidence? Ageing \& Society 23, 647-658.

Greenacre, M. (2010). Biplots in Practice. Fundacion BBVA Publication, Bilbao, Spain.

Grzywacz, J.G., \& Fuqua, J. MS. (2000) The Social Ecology of Health: Leverage Points and Linkages, Behavioral Medicine, 26:3, 101-115, DOI:

Hwang, T., Rabheru, K., Peisah, C., Reichman, W. and Ikeda, W. (2020). Loneliness and social isolation during the COVID-19 pandemic, International Psychogeriatrics, 32:10, 1217-1220

Jackson, D., Bradbury-Jones, C., Baptiste, D., Gelling, L., Morin, K., Neville, S., and Smith, G. D. (2020). Life in the pandemic: Some reflections on nursing in the context of COVID-19. Journal of Clinical

Nursing, 29(9-10)

Jenkins, E., McAuliffe, C., Hirania, S., Richardson, Thomson, K., McGuiness, L., Morrise, J., Kousolis, K. and Gadermann, A. A Portrait of the early and differential mental health impacts of the COVID-19 pandemic in Canada: Findings from the first wave of a nationally representative cross-sectional survey, Elsevier, https:/ / doi.org/10.1016/j.ypmed.2020.106333 retrieved June 25,2021

Uebersax, J.S. (2015). Introduction to the Tetrachoric and Polychoric Correlation Coefficients, retrieved from http://www.john-uebersax.com/stat/tetra.htm, 2021-06-28

Van Baarsen, B., Snijders, T. A., Smit, J. H., and Van Duijn, A. J. (2001). Lonely but not alone: emotional isolation and social isolation as two distinct dimensions of loneliness in older people. Educational and Psychological Measurement, 61, 1, 119-35. 